\title{
Harmonic Wavelet Envelope Method Applied in Railway Bearing Fault Diagnosis
}

\author{
Dechen Yao ${ }^{1 *}$, Limin Jia ${ }^{1}$, Min Li ${ }^{1}$, Yong Qin ${ }^{1}$, Wei Peng ${ }^{2}$, Guangwu Liu ${ }^{3}$ and Shaohuang Pang ${ }^{3}$ \\ ${ }^{1}$ State Key Laboratory of Rail Traffic Control and Safety, Beijing Jiaotong University Beijing, China \\ ${ }^{2}$ Beijing Materials handling research institute, Beijing, 100007, China \\ ${ }^{2}$ Guangzhou Metro.Guangzhou, 510030, China
}

Received 25 June 2012; Accepted 15 January 2013

\begin{abstract}
The working state of rolling bearing has an important influence on the operation of trains, directly related to the safety of train passengers. Therefore, it has great significance to conduct train bearing fault diagnosis. In this paper, based on harmonic wavelet envelope, a method for the fault diagnosis of railway bearings is proposed. First of all, the harmonic wavelet packet was used to translate vibration signal into time-scale representation. Then the decomposed signal was demodulated. Finally, through the analysis of the envelope spectrum, the bearing fault feature frequency was extracted. In order to verify the validity of diagnosis method, outer race fault bearing and ball fault bearing were tested. The test results show that the diagnosis method is effective and practical.
\end{abstract}

Keywords: Harmonic Wavelet, Envelope, Fault Diagnosis, Rolling Bearing

\section{Introduction}

The railway bearing vibration signals usually undergo a complex pathway before being collected by sensors. Because of the strong interference of outside, the required failure information is often submerged by the background noise, which is not conducive to signal feature extraction of the fault signal. Especially some early failure of the bearing, whose signal energy is small and the signal-to-noise ratio is low, making the signal processing and fault identification more difficult.

The vibration signal of the fault bearing is a non-stationary signal. Traditional spectrum analysis method can't effectively deal with non-stationary signals, For example, the FFT is used to analyze and observe the signal amplitude difference in frequency domain for damage diagnosis, and it is applicable to stationary signal feature extraction. Most practical dyadic wavelet is constructed by multi-resolution analysis of the two-scale equation, which usually hasn't clear mathematical expressions. Moreover, when the number of wavelet coefficients is small, the shape of the wavelet is irregular. In this paper, Harmonic wavelet envelope method is proposed to conduct bearing fault diagnosis, and the study is supported by rolling bearing test, which has achieved satisfying results.

\section{Harmonic wavelet}

The harmonic wavelet, constructed by Cambridge University in 1993, is a complex wavelet with clear expression that has strict box-shaped spectrum and good filtering properties [1]. Frequency function $\Psi_{e}(\omega)$ and $\Psi_{o}(\omega)$ are given as:

*E-mail address: shmily137@sina.com ISSN: 1791-2377 @ 2013 Kavala Institute of Technology. All rights reserved.
$\Psi_{\varepsilon}(\omega)= \begin{cases}1 / 4 \pi & -4 \pi \leq \omega<-2 \pi \\ 0 & \text { Other }\end{cases}$

$\Psi_{o}(\omega)= \begin{cases}i / 4 \pi & -4 \pi \leq \omega<-2 \pi \\ -i / 4 \pi & 2 \pi \leq \omega<4 \pi \\ 0 & \text { Other }\end{cases}$

And $\Psi_{e}(\omega)$ and $\Psi_{o}(\omega)$ make up a complex function:

$\Psi(\omega)=\Psi_{e}(\omega)+i \Psi_{o}(\omega)$

Then, the time-domain expression of the harmonic wavelet is obtained as:

$\psi_{e}(t)=(\sin 4 \pi t-\sin 2 \pi t) / 2 \pi t$

$\psi_{o}(t)=-(\cos 4 \pi t-\cos 2 \pi t) / 2 \pi t$

Thus, the time-domain expression of the complex function:

$\psi(t)=\psi_{e}(t)+i \psi_{o}(t)=\left(e^{i 4 \pi t}-e^{i 2 \pi t}\right) / i 2 \pi t$

The real part and imaginary part are shown in Fig. 1 and Fig. 2: 


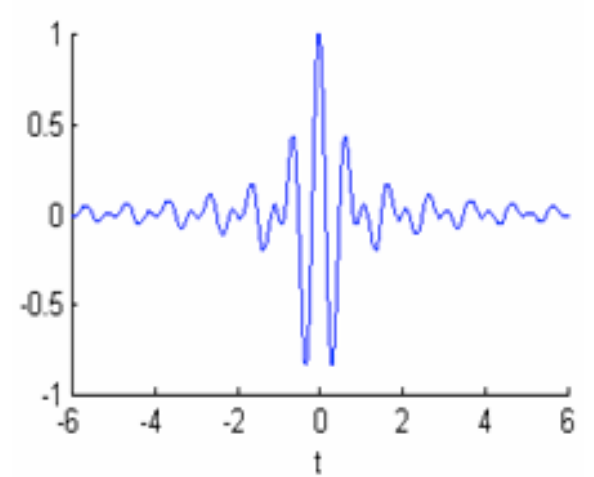

Fig. 1. The real part of harmonic wavelet



Fig. 2. The imaginary part of harmonic wavelet

Based on the formula (1) to (3), the Fourier transform of the harmonic wavelet is:

$\Psi(\omega)= \begin{cases}1 / 2 \pi & 2 \pi \leq \omega<4 \pi \\ 0 & \text { Other }\end{cases}$

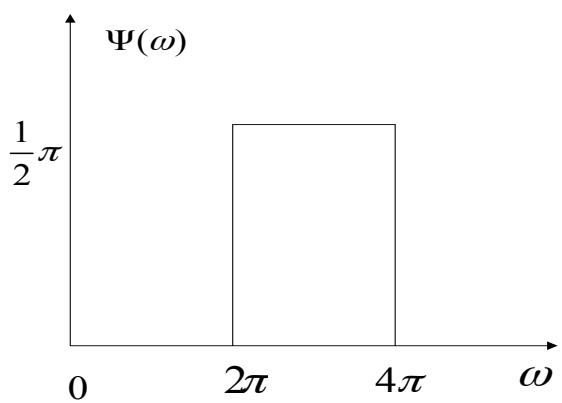

Fig. 3. The frequency spectrum of harmonic wavelet

The frequency spectrum of harmonic wavelet is shown in Fig. 3. It can be seen that the frequency spectrum of harmonic wavelet has the characteristics of the box and excellent compact support features [2].

Binary telescopic pan coefficient of harmonic wavelet $\Psi(\omega)$ can be conducted by using variable $2^{j} t-k(j, k \in Z)$ instead variable $t$ in the formula (6):

$\psi\left(2^{j} t-k\right)=\left(e^{i 4 \pi\left(2^{j} t-k\right)}-e^{i 2 \pi\left(2^{j} t-k\right)}\right) / i 2 \pi\left(2^{j} t-k\right)$

It can be seen that the shape of the harmonic wavelet is unchanged, but the scale is compressed in the horizontal direction, and the position is transmitted in the new scale of $k$ units [3].
Telescopic pan functions family of harmonic wavelet can constitute an orthonormal system. Signals can be decomposed into different frequency bands which are mutually independent by using harmonic wavelet as basis function without omission and overlap decomposition.

Introduce the positive real number $m, n(m<n)$, consider the band $\omega=[m 2 \pi, n 2 \pi]$, formula (7) can be generalized as:

$\Psi_{m, n}(\omega)=\left\{\begin{array}{cl}1 /(n-m) 2 \pi & m 2 \pi \leq \omega<n 2 \pi \\ 0 & \text { Other }\end{array}\right.$

Therefore, the corresponding wavelet is:

$\psi_{m, n}(t)=\left(e^{i 2 \pi n t}-e^{i 2 \pi m t}\right) / 2 \pi(n-m) t$

The value of $m, n$ is flexible, which can be a nonnegative integer value, and the value of $m, n$ can be an integer value as long as $m<n$, without limitation such as $n=2 m$. This greatly improves the flexibility when harmonic wavelet selects the analysis frequency band [4].

If the harmonic wavelet is translated by the step size $\frac{k}{n-m}(k \in z)$, then the formula (10) will become:

$\psi_{m, n}\left(t-\frac{k}{n-m}\right)=\frac{e^{i 2 \pi n\left(t-\frac{k}{n-m}\right)}-e^{i 2 \pi m\left(t-\frac{k}{n-m}\right)}}{2 \pi(n-m)\left(t-\frac{k}{n-m}\right)}$

So the analysis frequency bandwidth of harmonic wavelet is $(n-m) 2 \pi$, the analysis time center is $t=\frac{k}{n-m}$.

\section{Harmonic wavelet packet transform}

The harmonic wavelet can be decomposed by adjusting its parameters $m$ and $n$, so the good filtering function of harmonic wavelet can be applied to wavelet packet decomposition, and it does not reduce the number of signal points in the decomposition, ensuring the frequency resolution of the decomposed signal. Meanwhile, as harmonic wavelet has a phase preserving function, which does not change the phase of each harmonic component. So the harmonic wavelet can also achieve the band-stop filter and noise reduction by signal reconstruction after the decomposition.

Define $j$ as the number of the decomposition layers of harmonic wavelet packet; define $2^{j}$ as the number of subbands in the signal decomposition, define $f_{b}$ as the bandwidth of each sub-band; define $f_{k}$ as the highest analysis cutoff frequency of signal.

$$
f_{b}=f_{h} / 2^{j}
$$


Because the bandwidth of harmonic wavelet is $2 \pi(n-m)$, the wavelet scale parameter $m, n(m<n)$ must satisfy the following conditions:

$$
2 \pi f_{b}=2 \pi(n-m)
$$

Thus, the harmonic wavelet packet coefficient can be obtained by harmonic wavelet transform, that is:

$$
W P_{x}(j, i, k)=W_{x}(m, n, k)
$$

Wherein, $j$ is the number of the decomposition layers; $i$ is the band label; $k$ is the serial number of the harmonic wavelet packet coefficients. The following is a specific formula to determine the parameters $m, n(m<n)$ :

$$
m=i \times f_{b}=i \times \frac{f_{h}}{2^{j}}
$$

$n=(i+1) \times f_{b}=(i+1) \times \frac{f_{h}}{2^{j}}, i=0,1, \mathrm{~L}, 2^{j}-1$

Determine $j$, the upper and lower limit of the frequency band to be analyzed. Then wavelet coefficient can be obtained after the circular convolution operation, namely the decomposition of harmonic wavelet packet can be realized in the corresponding band [5]. The order of each node in the four-layer decomposition of harmonic wavelet packet is shown in Tab. 1.

\section{Signal envelope demodulation}

The envelope demodulation is an important technology to deal with the AM-FM signal, which can separate the bearing fault information from the complex modulation vibration signals. In this paper, the envelope demodulation of signals is realized by Hilbert transform.

Signal $g(t)$ becomes $\hat{g}(t)$ by Hilbert transform, and the mathematical expression is:

$$
\hat{g}(t)=\frac{1}{\pi} P \int_{-\infty}^{+\infty} \frac{g(\tau)}{t-\tau} d \tau
$$

$$
\hat{g}(t)=\frac{1}{\pi t} \times g(t)
$$

Wherein, $P$ is the Cauchy principal value. For simplicity, the value of $P$ is 1 .According to the above formula, Hilbert transform of the signal is the convolution of original signal in the time domain [6]. The convolution in the time domain is equivalent to the product in the frequency domain [7].

Hilbert transform is the basis of generating analytical signal. The method determined Hilbert transform pair is shown in Fig. 4, which uses the convolution theorem in the frequency domain. Original signal $g(t)$ and its Hilbert transform pair $\hat{g}(t)$ constitute the real part and the imaginary part of the analytical signal respectively [8]:

Tab. 1. Four layers decomposition of wavelet packet $\quad \hat{g}(t)$

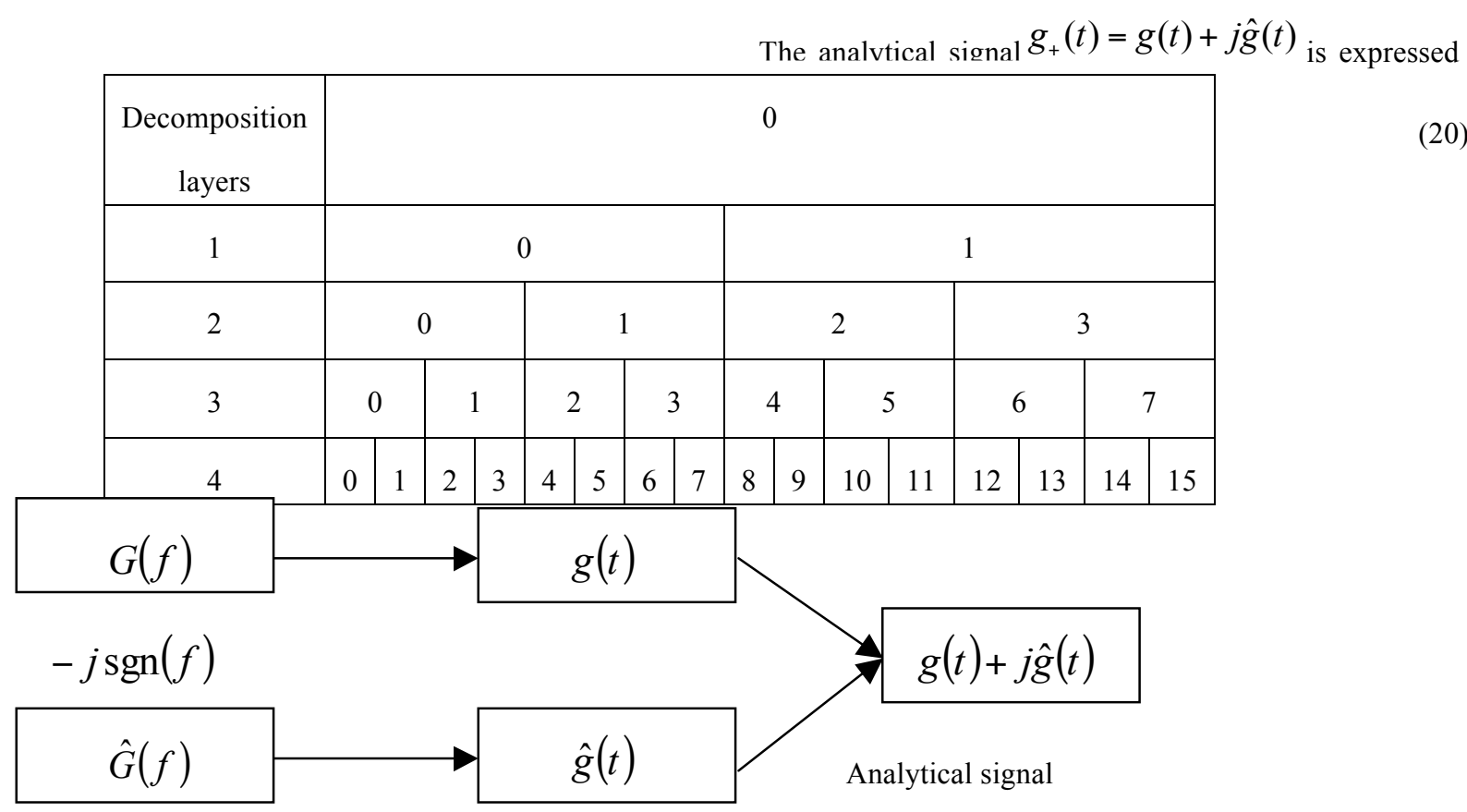

Fig. 4. Determination method of Hilbert transforms pair 
Wherein,

$$
A(t)=\sqrt{g^{2}(t)+\hat{g}^{2}(t)} \varphi(t)=\arctan \left|\frac{g(t)}{\hat{g}(t)}\right|
$$

The amplitude $A(t)$ is the envelope of the signal $g(t)$.

\section{Experimental study of harmonic wavelet envelope}

In this paper, fault diagnosis of rolling bearing based on Harmonic wavelet envelope is studied. Firstly, the frequency subdivision of train bearing fault signal is realized through the decomposition of harmonic wavelet packet. Then the decomposed signal is envelope demodulated by Hilbert transform. Finally, the type of fault can be identified by extracting characteristic frequency in the spectrum analysis. The following test of rolling bearing is based on this method.

In this test, outer race fault bearing is used and bearing speed is set to 1500RPM aimed to be as close as possible to the subway cars bearing speed. The sampling frequency is set to $10 \mathrm{kHz}$. The theoretical characteristic frequency of outer race fault is set to $117.8 \mathrm{~Hz}$.

Oscillogram of original signal of the outer race fault bearing is shown in Fig. 5. Modulation signal can be seen from Fig. 5, so bearing failure is preliminary recognized, which, however, could not be accurately positioned. Spectrogram of original signal is shown in Fig. 6. It can be roughly estimated from Fig. 6 that the value of carrier frequency center is set to $2300 \mathrm{~Hz}$, the bandwidth is set to $625 \mathrm{~Hz}$.

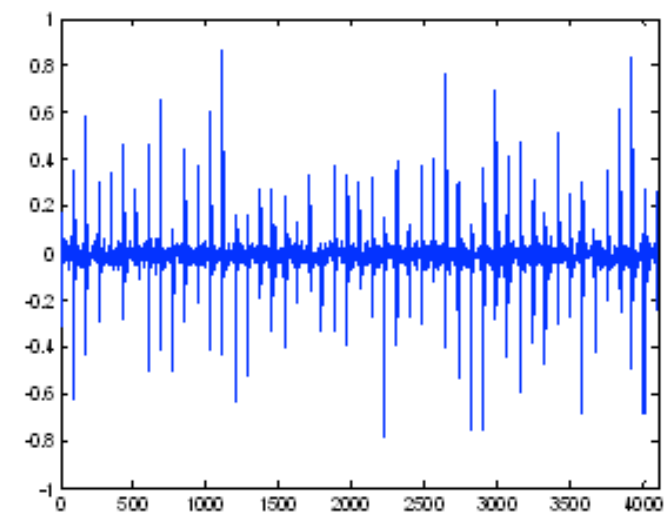

Fig. 5. Waveform of original signal

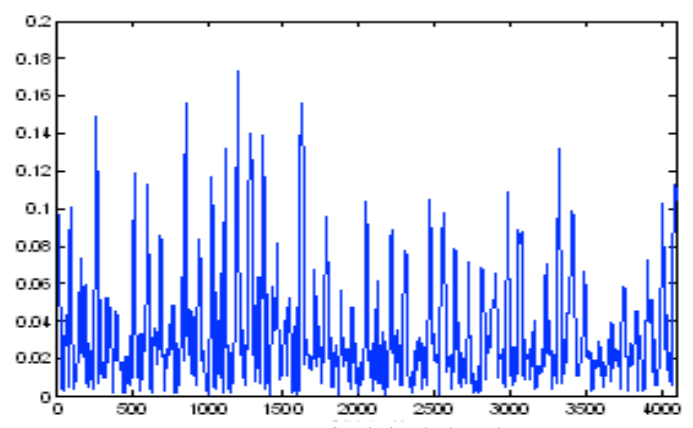

Fig. 6. Frequency spectrum of original signal

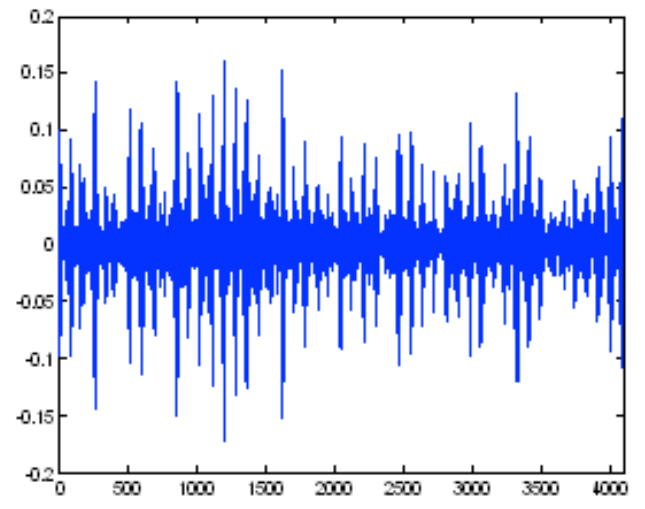

Fig. 7. The signal of node $(3,3)$ after the decomposition

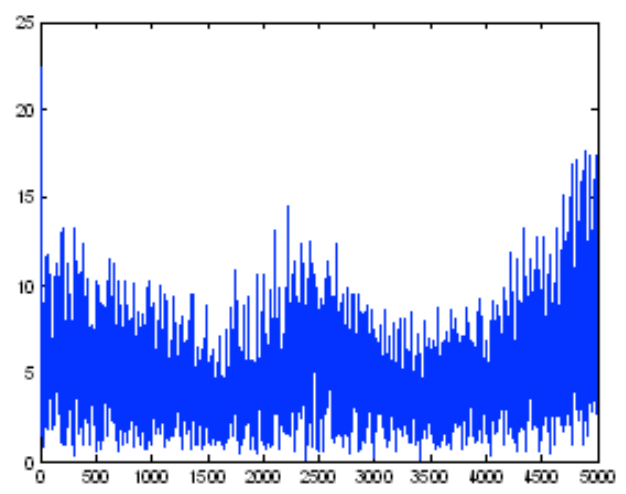

Fig. 8. Envelope signal

Decompose the fault signal through three-layer harmonic wavelet packet. The frequency range from $1875 \mathrm{~Hz}$ to 2500 $\mathrm{Hz}$ of node $(3,3)$ is shown in Fig. 7. The envelope signal after envelope demodulation of this node is shown in Fig. 8. Envelope spectrum of this node is shown in Fig. 9. Three distinct peaks can be clearly recognized from the figure, which are respectively $117.2 \mathrm{~Hz}, 234.4 \mathrm{~Hz}$ and $351.6 \mathrm{~Hz}$. Wherein, $117.2 \mathrm{~Hz}$ corresponds to the theoretical characteristic frequency $117.2 \mathrm{~Hz}$ of the outer race fault, so failure of outer race bearing is confirmed. $234.4 \mathrm{~Hz}$ and $351.6 \mathrm{~Hz}$ is, respectively, for its frequency doubling and frequency tripling.

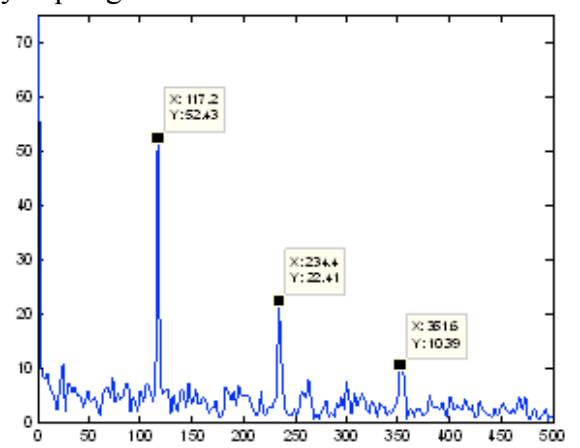

Fig. 9. Envelope spectrum

It can be obtained by comparing Fig. 6 and Fig. 9 that direct FFT transform for fault signal cannot distinguish the outer race ring failure. Decompose the wavelet through harmonic wavelet, and then extract envelope signal of the node at carrier frequency center, finally outer race fault can be diagnosed effectively through spectrum analysis. 


\section{Conclusions}

As can be seen by the above studies, decompose the frequency band through the harmonic wavelet packet, which can be regarded as the prefiltering of envelope demodulation. Then conduct envelope demodulation through the Hilbert transform and obtain the acceleration envelope spectrum of bearing fault signal. Weak fault signals can be extracted effectively by finding the modulation frequency through spectrum analysis, which represents failure characteristics. It has great significance in the diagnosis of subway train bearing early fault.

\section{Acknowledgements}

This paper was supported by the National Key Technology R\&D Program in the 11th Five year Plan of china (2011BAG01B05), the State Key Laboratory of Rail Traffic Control and Safety (No. RCS2010ZZ002), Beijing Jiaotong University and Doctor Foundation of Shandong Province (BS2011DX008).

\section{References}

1. Wu Xiao and Ji Guoyi, 2010. Applying harmonic wavelet packet detecting weak signal[J]. Electronic measurement technology, 33(6): $1-3$

2. E. D. Newland, 1999. Ridge and Phase Identification in the Frequency Analysis of Transient Signals by Harmonic wavelets[J]. ASME Journal of Vibration and Acoustics., 121:149-155

3. Li Yourong, 2008. Fault diagnosis of low-speed, high-load bearing based on generalized harmonic wavelet[J]. Journal of Wuhan University of Science and Technology (Natural Science Edition)., 31(1): $1-5$

4. Liu Xiaofeng and Peng Yongjin, 2011. Harmonic wavelet demodulation for gearbox fault diagnosis[J]. Journal of Chongqing University(Natural Science Edition)., 34(1):15-19
5. Zhang Jiafan, 2008. Envelope Demodulation Analysis Methods of Vibration Signals: Research and Application[D].Hubei. Wuhan University of Technology.

6. Han Yefeng, and Zhong Tao, 2010. Rolling bearing fault diagnosis based on envelope spectrum[J]. Mechanical Research \& Application., (4): 118-119

7. Zhang Xusheng and Zhu Yisheng, 1997. The methods of extracting signal envelope from Hilbert transform to wavelet transform[J]. Journal of Electronics and Information Technology., 19(1):120-123

8. Wu Yahui and Chen Donghai, 2009. Fault Diagnosis of Mine Gear Box based on the Wavelet Packet and Hilbert Demodulation Spectrum[J]. Journal of Mechanical Transmission.,32(2):72-89 